The International Conference : Cities' Identity Through Architecture and Arts (CITAA)

\title{
Interior Architectural Elements that Affect Human Psychology and Behavior
}

\author{
DOI: $10.21625 /$ archive.v1i1.112
}

\section{Heba-Talla Hamdy Mahmoud ${ }^{1}$}

${ }^{1}$ Lecturer of interior architecture Decor department Faculty of Fine Arts Mansoura University

\begin{abstract}
Keywords
Awareness; Identity; Privacy;

Functionality; flexibility Safety;

Health concern; Accessibility;

Open space; Aesthetic;
\end{abstract}

\begin{abstract}
This research will inspect factors with higher impact that are predicted to be more influential in the relation between architecture, interior architectural design and the psychological status of residents and users. The level of awareness about the importance of this relation is the basic introductory factor. Identity, privacy and safety impacts, health concerns, accessibility degree, open spaces feature, aesthetic sense are the main parts of the research. Most parts consist of two divisions. The first identifies the nature of each factor. The second recognizes the important architectural consideration needed to realize the psychological condition of residents and users. The research aims to increase the concern about the importance of the interaction between interior architectural design and human psychological behavior. An introduction of a group of important consideration can be used to help designers choose and apply a suitable interior architectural design that match psychological needs through sound relations between architecture, interior architecture and the psychological status of residents and users.
\end{abstract}

\section{Introduction}

The psychological attitude of a human is affected by the design of interior architecture through various aspects. every person receives, perceives and responds in different way, this is due to physical and psychological differences in addition to the differences in personal experience. Culture, physical status, age, education level, gender, socioeconomic class and ambitions are factors with special concerns that shaping occupants' needs. The interaction between interior architecture and the psychological condition is engaged with both humanitarian characteristics and the interior architecture approach of design. "Architecture leans towards programming i.e. determining needs and proposing a draft and finally suggesting a suitable location in accordance with needs and building of the site. behavioral sciences on the other hand are concerned with how environment is used in terms of consistency with needs of society" (Razjoyan, 1996)."The mental and psychological effects of architectural frames on human beings have been considered from the early shelters to today's modern structure. Since the human behavior is performed in defined spaces, it is necessary to design the physical space based on people’s behavioral characteristics". (Tabaeian, 2011). The focal point of the architectural side is the background and capability of the designer to create a comprehensive vision with respect to the psychological intervention of all parameters involved in his design and to identify the considerations must be taken into account. "One of the essential roles of architecture is to provide built environments that sustain the occupants' psychological well-being. This role is made even more important because, in modern society, more than $70 \%$ of a person's lifespan is spent indoors" (Kim, 1998).

\section{Research methodology}

The methodology of this research is generally based on a descriptive and interpretive approach. An initial survey to investgate the relation between architecture, interior architectural design and the psychological status of residents and 
users was conducted. Questions included the following: What is the backgrounds and scopes of architecture, interior architecture designer needed for the designe to recognize in order to create a right vision? What are the most effective parameters that impact this relationship? This research selects the most effective factors (identity, privacy, functionality and felexability, safety and health concern, accessibility, open space, aesthetic) that interact with the relation between architecture, interior architectural design and the psychological status of residents and users. Other questions include: What are the main distinctive considerations that should be taken in to account to handle these factors? Many data sources included books, journals, conferences, and specific official internet sites.

\section{Interior architecture design psycological awareness}

The achievement of interior architectural design, with awareness of the psychological impacts on residents, needs a wide scope of knowledge about the different dimensions and circumstances that should be taken into account Figure 3-1. "As practitioners, we solve the design problems for humans who use and inhabit the space by considering their needs, whether functional, social, psychological or environmental by understanding how to use research to help identify and clarify the relationship between human behavior and the built environment" (Perolini, 2006). Different methods of investigating and different approaches were used to recognize and shape the complex network of relations between architecture, interior architecture design and the psychological status of residents and users. "A useful model for seeing the scope of available environment -behavior information Figure3-2m first proposed by the architectural psychologist Irwin Altman ${ }^{1}$ includes three main components: environment-behavior phenomena, user groups, and settings" (Burris, 2014). A different approach to identify the wide scope of comfort in homes through a multi-dimensional survey was composed of three main proposed dimensions (Meaning of Comfort at Home, Home Environment, and Comfort), Figure3-3.

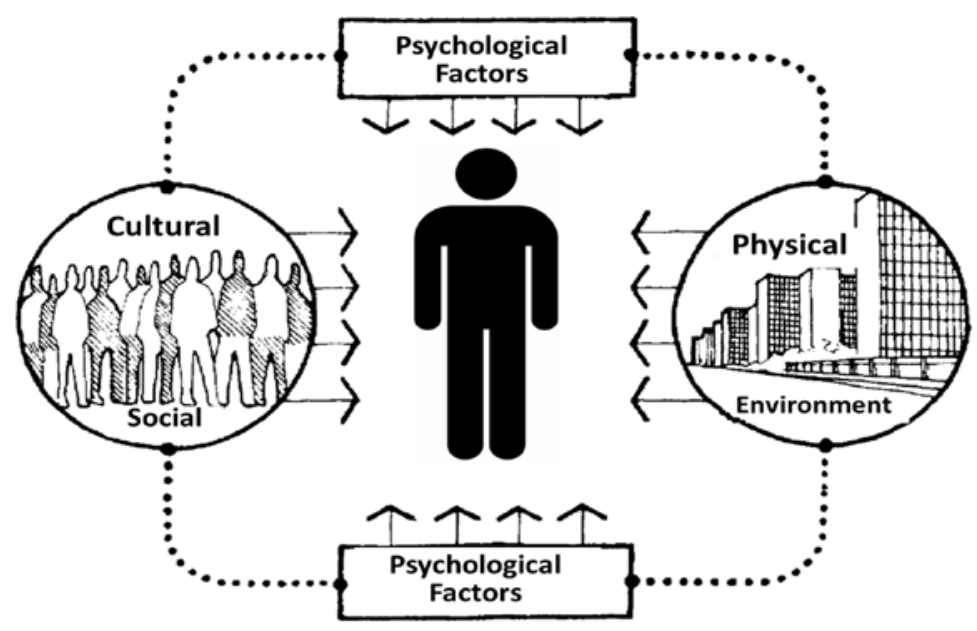

Figure 3-1: Factors impacts human psychology (Moore 1979).

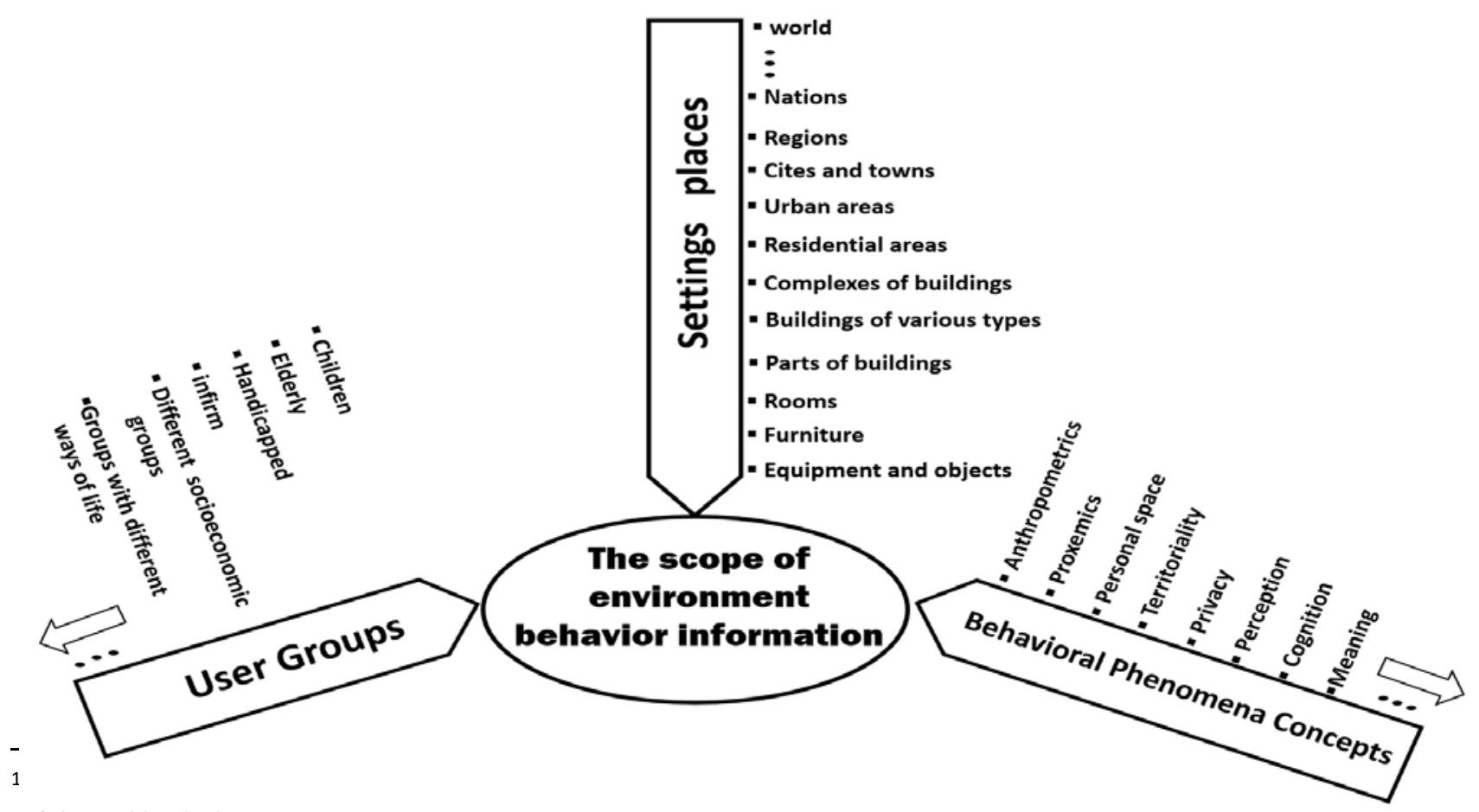

articles and book chapters 
Figure 3-2: The scope of environment -behavior information. (Snyder JC 1797)

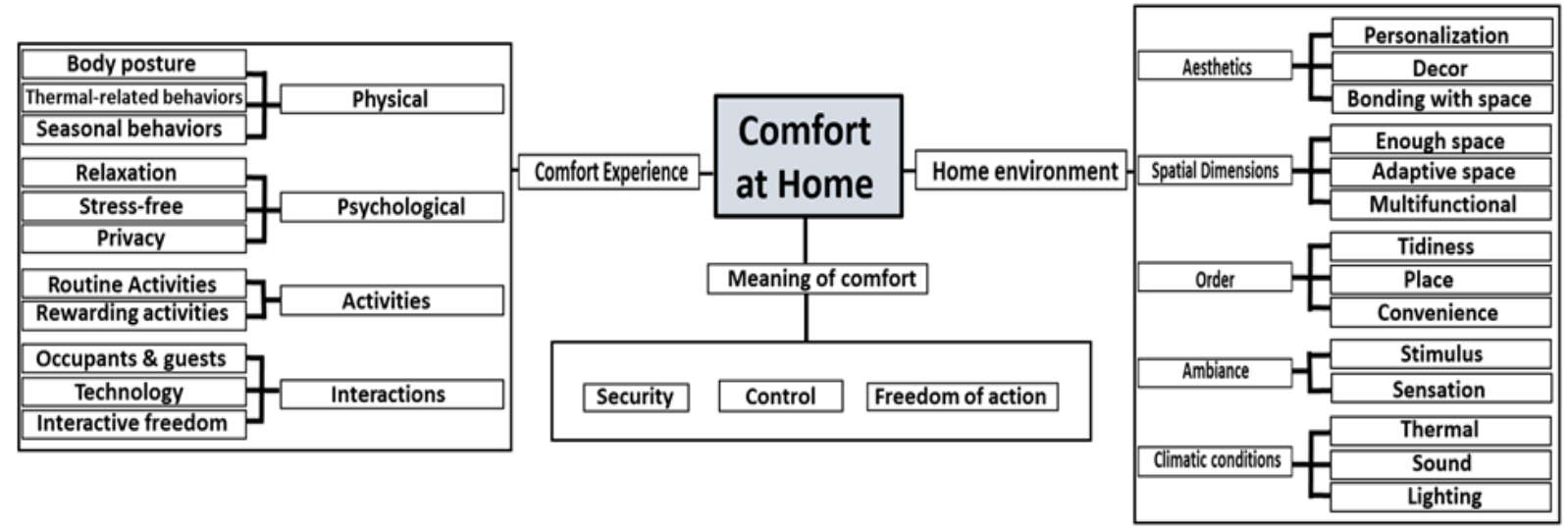

Figure 3-3: Dimensions and sub-dimensions of home comfort (Burris 2014)

\section{The psychological impact of identity}

Human perceptions of the space they live in, work, or spend time in are directly impacted by the reflection of personal, socil, and cultural identities on these places. It is convenient that places remind us of the main features of our identity, "there is a complex interaction between a person and a space. The person defines the space, the space defines the person; the person gives meaning to the space, the space gives meaning to the person. In other words, there is a complex and bilateral interaction between the person and the space in its cultural, psychological, economic and physical dimensions" (Ayalp, 2012). The sense of psychological identity about the architecture of space grows when we start to identify ourselves with places. "The field of psychology and the social sciences of architecture, emphasizing the influence of the physical environment on identity and self-perception" (Hauge, 2007). The psychological identity sense and its interaction with interior architecture occurs in both larger scale domains such as culture, religion, nation, city, gender, social roles or social class and in smaller domains such as neighborhood, family, homes and rooms. Generally, we can say that humans have always showed their dedication to their roots. It can be said that every civilization or ethnic group offers a specific architectural identity in accordance with their cultures and traditions at different times. The psychological identity about space is affected by the international architectural trends due to globalization and its nature as a standardizing force in economics, politics, culture, and consequently in architecture. The architucture of Hassan Fathy is considered a remarkable style of the interaction between authenticity in the orientation of identity and contemporary architecture. "The invaluable legacy of Hassan Fathy constitutes inspiration for younger architects whose prime concerns are to preserve cultural identity in architecture while providing appropriate and affordable shelters for the disinherited of this world" (Richards, 1985). It essential to recognize that identity does not depend on the composition and form of physical space only, but it is linked to the concept of meaning and sense. There are numerous factors that shape identity and selfexpression such as the structure of family and relatives, and child-raising methods.

\section{Interior architecture consideration for identity}

Architecture and interior architecture designers should maintain the identity of peoples and communities and respect their daily routine of life. The appropriate implementation of design aims to achieve the present needs of users and make it suitable for future generations."People feel comfortable in the places that are concurrent with their place identities. Moreover, with reflecting true cultural identities, designer can take part in sustainability of cultural value. The typical features that are creating the image are the basic elements in designing new images" (Ayalp, 2012). Figure 4-1 is an interior space in Japan that reflects japanese interior architectural concepts and their identity. Figure 4-2 is a facade of building in Yemen, it represents the character of Yemeni architecture as a remarkable identity. The following are examples of architecture and interior architectural considerations with psychological impacts related to identity:

- The design should demonstrate the background and motives of residents.

- The design should demonstrate the structure of religions, cites, families and neighborhoods.

- Usage of local materials and technique such as plaster, stone and marble.

- Architectural treatments of openings, windows and doors, especially in the main entrance framing and decoration.

- The diversity and richness of arts and architectural treatments that belong to the local and national tradition.

- Social and special niches, signs and icons.

- "The level of modern influence by western and international style concepts in comparison to the accustomed norm of the traditional architecture of the region can be categorized as follows:

- Copying the traditional architecture of the region without any modifications with no change. 
- Minor changes: Partial change of system elements

- Adaptations: Mixing the source with new elements

- Major changes: Changing the system relation

- Total changes: Altering the system rules and regulations" (Baper, 2010).

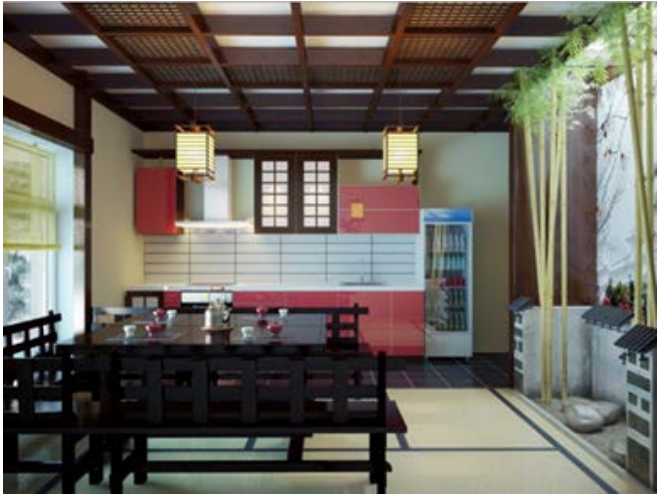

Figure 4-1: Interior space pattern in Japan

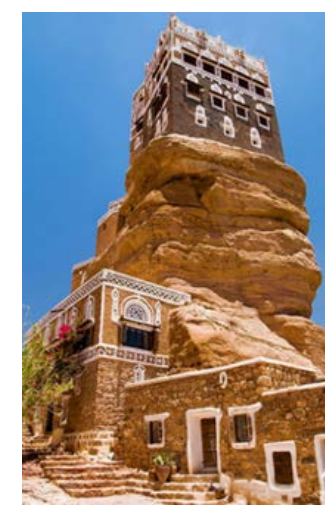

Figure 4-2: Building's façade in Yemen

\section{The psychological impact of privacy}

Privacy in general is the right of individuals, groups, organization, and institutions to determine for themselves when, how and to what extent information about them is communicated to others. In a public space, there is no restriction of communication, while isolated spaces completely constrain all types of communication. In between there are intermediate levels of privacy. Space privacy is considered one of the most important types of overall privacy. People deal with the concept of space privacy with special concerns, they feel discomfort, anger and anxiety when their space privacy is exposed beyond their desires. "According to environmental psychology, each person is realized and perceived through an invisible shelter or a series of shelters surrounding his body, Figure 5-1. These personal protective spheres, by which privacy is controlled, vary from person to person and from culture to culture. They also differ from period to period as society and social bonds are continually transformed and reconstructed. Hall defines accordingly four such spheres; intimate, personal, private and public. When the most intimate of these private areas is intruded by other individuals, the person starts to act defensively or to say at least extraordinarily. A typical example of the above fact is indicated by the abnormal behavior of people when standing in an elevator" (Hall, 1969).

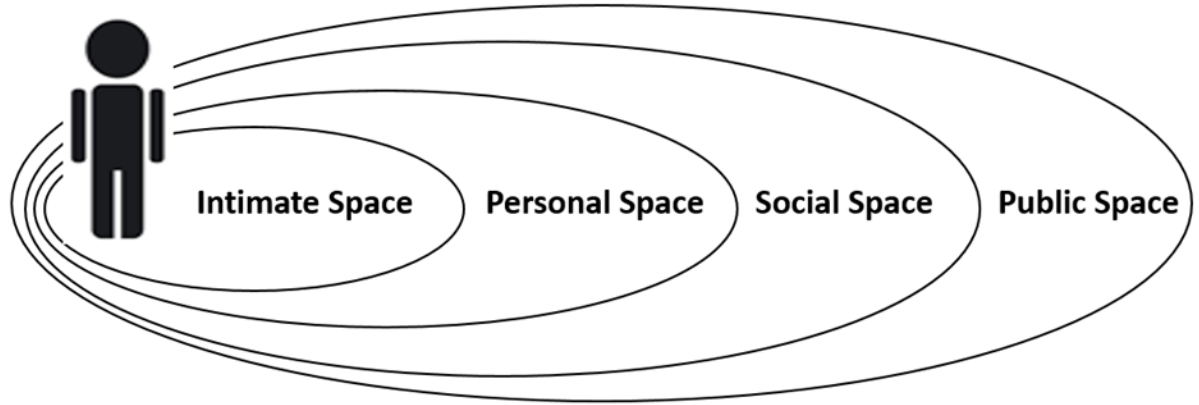

Figure 5-1: The main four categories of space, the hidden dimension 1990

\section{Interior architectural considerations for privacy}

The central aspect of achieving privacy is how to find a balance between privacy and social relationships. Accessibility, visibility, proximity, vocals and olfactory are the main parameters of privacy interaction with the human psychology. Each parameter requires numerous suggestions of considerations, nevertheless the general guidance can be performed through using space sizing and organizing with vertical, horizontal boundaries, partitions and conversation shields. The degree of light and illumination distribution with direct and indirect lighting needs to be controlled. Using appropriate types of sound-blocking, soundproofing, curtains, floor walls, furniture, and equipment. "The careful design of enclosed space enhances privacy through auditory isolation, dimmed lighting, and a semi-transparent divider between penitent and priest, stimulates a moment of reflection, serenity, and honesty" (Petermans. A, 2014)."According to the degrees of proximity, there are four categories of privacy with specific consideration for each...Intimate distance of eighteen inches or less, the presence of another person is unmistakable and may at times be overwhelming because of the greatly steppedup sensory inputs. In its close phase (6 inches or less) intimate distance lends itself primarily to nonverbal communication. This is distance is usually reserved for very close friends and family. Personal distance from $11 / 2$ to 4 feet can be thought 
of as a small protective sphere or bubble that an organism maintains between itself and others. Social distance ranges from 4 to 12 feet, it's a psychological distance, one at which the animal apparently begins to feel anxious when he exceeds its limits. We can think of it as a hidden band that contains the group. Public distance is the largest of the zones and it exists only in human relationships. At the close phase (12 to 25 feet), a more formal style of language and a louder voice is required" (Hall, 1969). The following are examples of architecture and interior architectural considerations with psychological impacts related to privacy:

- Public and private spaces should be clearly defined by means of applicable well-defined boundaries,

- The degree of interrelationship and connectivity with adjacent spaces.

- The type of mass zone and courtyard which is surrounding the space.

- The difference between the street level and the ground floor level.

- Number of entrances, is there one entrance or more.

- The implementation of privacy practices through a monitoring service.

- The type of transitional space behind each access and the degree of exposure to inside spaces.

- The existence of a part of, or an entire floor below ground level (basement).

- Type of walls and insulation partition.

- The type of main movement system, even if it's radial, is linked to one axis or another

- The style, size and direction of openness (windows, doors) regarding the overall size and orientation.

- Kitchen design and type (closed or open plan kitchen).

- The orientation of private rooms' doors and openings toward the open and living spaces.

- Roof type, degree of exposure to inside spaces.

\section{The psychological impact of functionality and flexability}

The satisfaction and psychological status of users and residents is directly linked to a well-planned and designed feature that makes spaces more usable and beneficial. Functionality consideration encourages people to live and work effectively. The psychological and physical comfort of residents is linked to the degree of feasibility and flexibility of the design. The possibilities of flexible design to change the shape and size of internal space (Figure 6-1), and the impact of feasible functions of interior design in addition to the using of the space as integrated environment make our interior more livable. "Interior architecture can have to function as a platform for happiness and human flourishing, as a combination of positive feelings and optimal human functioning. It is clear that design can function as a direct source of pleasure or facilitate pleasurable activities. Such a vision incorporates a view on interior architecture as an activating and dynamic platform that facilitates the occurring of meaningful activities for its inhabitants" (Petermans, 2014). "User flexibility is an important design objective of modern dwelling units. The provision of various possible spatial configurations of interior space sub-division. Created within defined dwelling space, is a natural necessity resulting from altering dwelling needs over life span, change in living standards, use of modern household appliances and variation in life style and fashion. The majority of dwellers face the necessity of enlarging their dwelling space as a result of familial growth. Conducting work from home and enjoying hobby activity. This process could later be reversed when grown up children leave home and the need for space is decreased. The possibility to move to another dwelling unit is not always attainable as a result of financial situation. Social factors and children education. User flexibility is. Therefore, called for to provide dwellers with a variety of possibilities to sub-divide given dwelling space and use it in the best possible manner" (Karni, 2000).
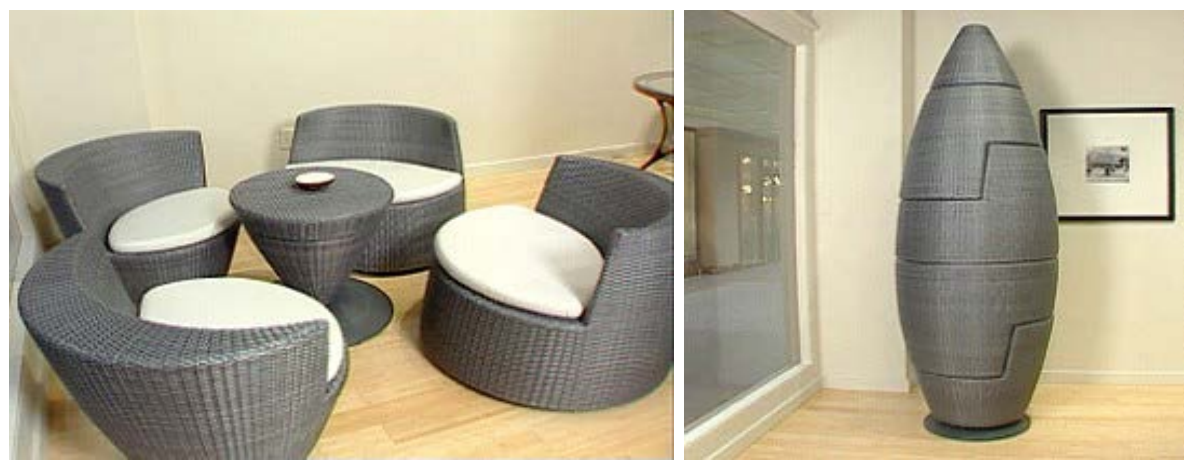

Figure 6-1: The flexibility of furniture design and space usage

\section{Interior architectural considerations for functionality and flexability}

Maximizing the functionality and flexibility of space requires that some important questions be answered by the designer. Who will be there? What is their lifestyle throughout the course of time? What features and facilities are most important to them? The differentiation between the multiple building types, whatever residences, workplaces, healthcare facilities, learning environments, and retail spaces is a vital element of functionality and flexibility considerations. From the financial point of view, smart functional characteristics are considered an effective way for reducing expenditure 
consuming less products, space and energy. Subsequently, this will be reflected on the psychological state of peoples. "Maximum \& optimum utilization of the interior space requires flexible design of furniture in the space. Many furniture pieces are designed using folding patterns or drawers included with mechanical or electrical features and this furniture can the interior space very efficient through ergonomic and human fitness" (Emamgholi, 2011). The following are examples of architecture and interior architectural considerations with psychological impacts related to functionality and flexibility:

- Apply appropriate design for windows and doors to adopt all diverse physical abilities.

- Adopt the concept of one space that can reflect different social functions.

- Chose appropriate colors for switches and sockets that contrast with its background to be reached easily.

- Equipment such as fans, air conditioning or the fuse box are easy to locate and operate.

- Design the living room to accommodate various functions and sizes of group activities.

- Sufficient lighting in the kitchen, over bathroom, hand-basin and work areas.

- Select a wall system that offers a complete range of options from private space to open plans

- In public buildings, the lobby should be broken down into smaller areas, where groups can gather.

- Make multiple furniture configurations available.

- Ensure that the design provides contemporary technology requirements.

- Select durable floors, ceilings and walls finishing materials.

- Select durable furniture designs and materials.

- Ensure adequate cable interfaces with precise placements to offers appropriate degrees of flexibility.

- The kitchen design should incorporate work surfaces for both standing and seated use.

- Increase the size of desk tops surfaces and maximize vertical space.

\section{The psychological impact of safety and health concerns}

One of the main psychological concern of users and residents depends on having homes and settings that are safe and free from physical hazards. "The quality of housing conditions plays a decisive role in the health status of the residents. Many health problems are either directly or indirectly related to the building itself, because of the construction materials that were used and the equipment installed, or the size or design of the individual dwellings. Representing the spatial point of reference for each individual, the home also has a broad influence on the psychosocial and mental well-being by providing the basis for place attachment and identity as well as a last refuge from daily life. However, especially, for this mental dimension of housing satisfaction and the meaning of home to the resident, not much data on the relation between health and well-being, and subjective satisfaction, and housing perception are available" (Bonnefoy, 2007). Architects and interior architects share the responsibility with both occupants and administrative authorities with regards to safety and health considerations of occupants and users according to rules and instructions. The psychological effect of safety and health care increased when related to children, elder people and people with disabilities. Most Injuries such as fall burns, and poisonings usually occur at home due to improper architectural design. "There are two common strategies in building design that are employed to deal with the indoor environmental quality (IAQ). The first one is to improve the indoor air quality by increasing the ventilation rate, which in turn reduces air pollutant. The second is by reducing the source of pollution within and outside the building in order to reduce the introduction of pollutants in the indoor air" (Alhorr, 2016). "A home perceived as safe and intimate provides major psychosocial benefits. It represents a protected refuge from the outside world, enables the development of a sense of identity and attachment - as an individual or as a part of a family, and provides a space to be oneself. Any intrusion of external factors or stressors strongly limits this feeling of safety, intimacy, and control, thereby reducing the mental and social function of the home" (Kearns, 2000)."There are many indoor stressors (e.g. thermal factors, lighting aspects, moisture, mould, noise and vibration, radiation, chemical compounds, particulates) that can cause their effects additively or through complex interactions (synergistic or antagonistic). It has been shown that exposure to these stressors can cause both short-term and long-term effects" (Bluyssen, 2013).

\section{Interior architectural considerations for safety and health concerns}

Architects and interior architects have a wide range of options to apply safety and health concerns considerations by adapting their design to utilize the features of climate environmental conditions through controlling the degree of sun exposure, temperature, wind, humidity and veneration. They should be aware of the materials they choose for their design, such as glass, paints, fabric types. the strength of friction as slip resistance. They need to be careful about the causes of toxicity and pollution that affect indoor air quality for preventing any chemical contamination especially with all types of paints, fabric and wall floor coverings. They are responsible for applying codes, rules and standards. The following are examples of architecture and interior architectural considerations with psychological impacts related to health concerns:

- Ensure safety fence with secured access to the interior space.

- Signs should be useful with adequate size with right orientation and clear text and figures.

- Avoid sudden changes of level which could trip people, if they are present, it must be made clearly visible with contrasting colors.

- Convenient entrances, people should be able to safely enter and exit without obstruction. 
- Make the floor slip-resistant, non-reflective light, glare-free and easy to clean.

- Solid core construction for interior spaces doors.

- Enhance the levels of lighting where it's needed, it should also be easy to control and adjust.

- Enhance the implementation of safety practices with technical equipment such as intercom system and security cameras with monitoring response service.

- Stairs should be in appropriate width, right steps dimension, strong handrails and adequate lighting. (Figure 7-1)

- The appropriate distribution of appliances in accurate positions such as stoves and air conditioners.

- Install automatic smoke detector system.

- Choose comfortable and safe furniture with healthy design.

- Maximize the daylight availability through opening (windows - doors) dimensions, materials and design.

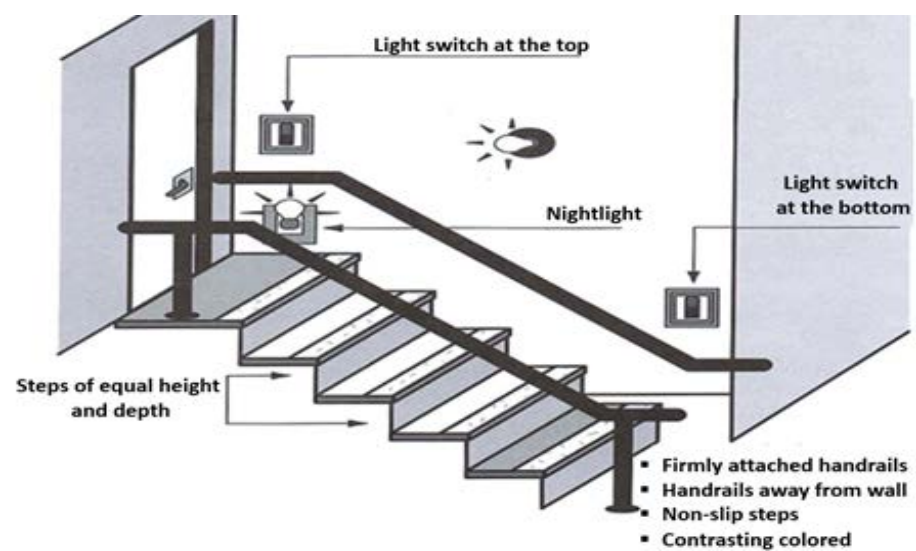

Figure 7-1: Stairs safety consideration.

\section{The psychological impact of accessibility and circulation}

Architecture circulation and interior architecture movement and accessibility is a continuous process that we exercise in our daily life. This process may be as easy as moving from one room to another or as difficult as trying to escape a building on fire. Efficient navigation through the exteriors and interiors of any space saves physical efforts, enhancing emotional status and introduce a primary impressions about the overall quality of the architectural design. Circulation difficulties may cause problems such as loss of time, decreased safety, or causes stress and discomfort. Accessibility and circulation tasks are affected by two major factors, the architecture and interior architectural design of the space and the degree of information clearness and accuracy. The impact of accessibility on physical and psychological conditions take further importance when related to public buildings, particularly public buildings with large complex facilities such as shopping centers, airports and hospitals. Spaces and buildings must be accessible to all people with special concern to children, old people and persons with disabilities.

\section{Interior architectural considerations for accessibility and circulation}

The designs of interior architectural accessibility and circulation vary according to space type, size, layout and user requirements. The design of these processes need wide scopes more than putting up signs or just design an entrance. All routes of both horizontal circulation elements and vertical circulation elements within any space or building should be as free as possiple of obstacles and they should be easy to distinguish. The following are examples of architecture and interior architectural considerations with psychological impacts related to accessibility and circulation:

- Approaches from streets toward a building or space should be leveled, clearly identified, and slip resistant. Figure8-1

- Entrances should be clearly defined and furnished with adequate light

- Use signs to identify the paths of horizontal and vertical circulation systems.

- Lobbies should accommodate visitors with waiting areas and privide information about the space.

- Halls and corridors should provide safe and adequate circulation between space units.

- Stairs to the upper level should be suitable for people with disabilities.

- Elevator should have adequate space in comparison with the volume of use.

- Elevator should be visible with wide-angle from entrance.

- Lighting plan should illuminate all space parts with suitable and adequate light.

- Let room's space design allow access to furniture, storage, windows and appliances. 


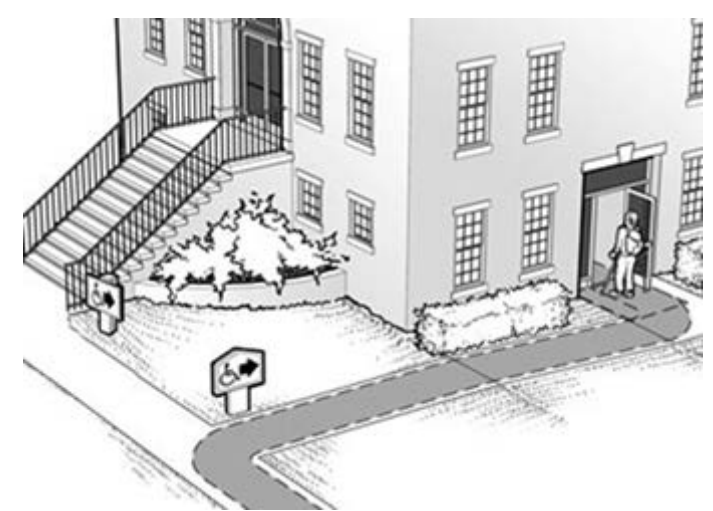

Figure 8-1: Approaches should be leveled, clearly identified, slip resistant

\section{The psychological impact of outdoor and open spaces}

The physiological studies about the outdoors and open spaces indicate that the natural environment can contribute to reducing anger, anxiety, and helps in relaxation management and improves the overall health outcome. Both visual access and being actually within a green space helps to improve the ability to focus and increase the level of alertness. Outdoors and green spaces encourage social contact, leisure and cultural activities by sharing activities with others and enhance the desire of knowledge. Outdoors and open spaces provide the availability of physical activity like walk and exercise. "Over the past several decades, research in a variety of fields such as workplaces, hospitals, urban environments, and experimental laboratories shows that contact with nature generates emotional, physiological, social, and cognitive benefits. Furthermore, the findings point consistently to the value of particular natural features such as large trees, flowers, gardens, and water. Studies also show that benefits of nature occur in many ways -- through direct contact (sitting in an outdoor garden), indirect contact (through a window view), and from simulations using nature decor such as posters or paintings" (Heerwagen, 2006).

\section{Interior architectural considerations for outdoors and open spaces}

Outdoor and open spaces are a broad term covering a wide range of residential and areas. It's important to recognize that the psychological consideration of inhabitant's usage of outdoor and open spaces in private spaces like houses and homes vary from those in public spaces. The designer needs to understand and evaluate the opportunities available for his or her space to utilize every positive option of environmental conditions and nature like sun, air, and greening.

- Create visual harmony between the indoor spaces and outdoors spaces.

- Appropriate fence design, with regards to form, height, and the degree of transparency with exterior spaces

- Ensure that entrances, openness and pathways enable people to move freely, Figure 9-1.

- Ground surfaces should be a mix of hard and soft surfaces according to the nature of use.

- Electrical and network interfaces should be available with easy to use potential technology.

- Design should be planned to minimize negative factors like exterior noise and smoke.

- Sufficient night-time lighting to enhance safety, security and ensure efficient usage of space.

- Design should be planned to enhance the visibility degree.

- Vegetation with colored flowers and leaves provide an attractive view.

- Design should provide multiple sitting areas and cooking facilities, Figure 9-2.

- The selection of outdoor furniture should depend on their strength, durability, beauty and appropriate formation.

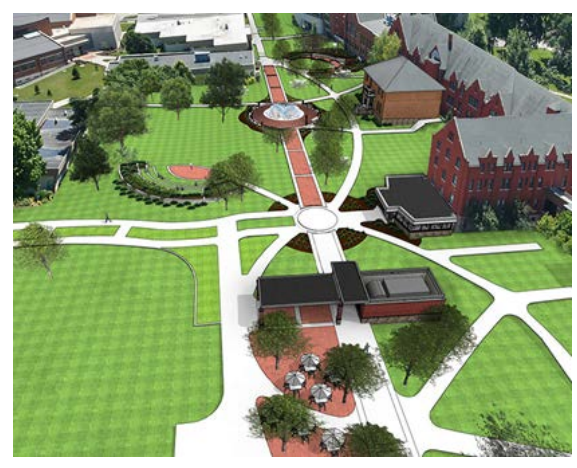

Figure 9-1: Pathways enable peoples move freely

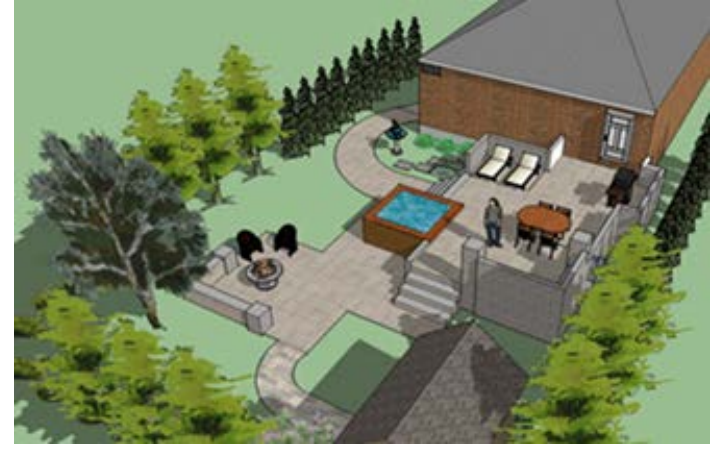

Figure9-2: Multiple sitting areas and cooking facilities 


\section{The psychological impact of the aesthetic aspect}

Aesthetics play an essential role in the emotional satisfaction of users and residents when it comes to the architecture and interior architectural design. It's directly associated with human pleasure and spirit. The success of interior architecture designers to reach the aesthetics of a space depends on the degree by which he or she understands human sensitivity to beauty and expression perception. "Settings and traditional towns are examples of pleasant atmospheres often arising from aesthetically rather uninteresting units. Such urban atmospheres are most often created by specific materiality, scale, rhythm, color or formal theme with variations"( Pallasmaa, 2014). Aesthetics are defined by each individual, each family, each group and each society. As a result, the aesthetical status of space will function properly when we investigate inhabitant's definitions of beauty and consider their vision. This investigation should include the foundation of local traditions, the present community and aspirations for future. "Aesthetic appreciation is both expressed in and influenced by the environment. To define aesthetic qualities, the designer needs to understand that the concept of beauty differs with time and place, purpose and context. Values captured under the label "aesthetic" can best be understood at a universally comprehensible level. These aspects of a design go beyond the functional and constructional concerns, and are associated with the specific way the design presents itself to the human senses" (Cheung, 1997).

\section{Interior architectural considerations for the aesthetic aspect}

Interior architectural designers express the aesthetics and beauty of a certain space through using the main factors that influence the implementation of aesthetics and beauty (form - texture - color - light). "Forms are constructed from points, lines, planes, surfaces and volumes that are made richer by texture, color and material. In combination, these elements of form create a design, and similarly all of these elements contribute to our perception of its meaning. The designer could be cast in the role of a communicator whose messages to the user concern the symbolic qualities of products. Just as a journalist creates informative messages from a vocabulary of terms, so could a designer be thought of as having a collection of forms at his disposal with which he creates arrangements that can be understood as a whole in their essential parts and that are usable by a receiver because of this communicated understanding" (Mothersill, 2014). Every surface has its texture which can be smooth or rough, glossy or unpolished, bumpy or flat. Textural contrast sensibility has its direct impact on the mind based on our memory of touching similar surfaces. The impression of color is categorized within the maximum impact of space factors that interact with the psychological mood. Light can be used to achieve emotional responses. We can produce certain moods such as restfulness, activity, warmth, and coolness through using lighting patterns of varying levels of illumination. The following are examples of architecture and interior architecture considerations with psychological impacts related to the aesthetic aspect:

- Create forms that express the user's meanings and preference.

- Maximize the use of natural lights in compatible balance with artificial light.

- Feasible utilization of modern materials that display a flowing and multilayered curves construction.

- Estimate the appropriate contrast between floor, walls and ceilings.

- Consider the flexibility and level of illumination for specific tasks.

- Understand the impact of each color on the human psychological mood.

- Choose color plans based on the analysis of color preferences of users and residents.

- Appropriate geometric lines and ornamental decorations.

- Display interesting artworks, photos and other personal items.

- Ensure convenient relations between the major pieces of furniture, walls, and floors regarding the size and balance.

- Find a balance between unity and variety, too much unity without variety is boring and too much variation without unity is confusing.

\section{CONCLUSION}

The scope of the mutual relation between interior architecture and human psychology is so wide, that is due to the multiple interaction with social, cultural, physical and environmental factors. Most studies about the interaction between interior architectural design and the psychological status of people are comparatively a contemporary approach, a detailed understanding still needs to be refined. The successful design for achieving welfare and happiness of interior architecture depends on how the designer finds a balance between the most dominant factors such as identity, privacy, safety, accessibility, functionality, flexibility, community interaction, and the provision of adequate space, should be given due weight. Ultimately the success of setting up interior architectural design with active psychological conception judged by how the design fulfills values, needs, preferences and satisfactions of users. The main question is, what are the designers' experience, qualifications and imagination about the various factors of interior architecture that affect human psychology and behavior. 


\section{REFRENCES:}

Adler, D. (1999). Metric handbook: planning and design data (Second ed.). Oxford: Educational and Professional Publishing Ltd. Alhorr, Y., Arif, M., Katafygiotou, M., Mazroei, A., Kaushik, A., \& Elsarrag, E. (2016). Impact of indoor environmental quality on occupant well-being and comfort. International Journal of Sustainable Built Environment, 5, 4-7.

Alitajer, S., \& Nojoumi, G. (2016). Privacy at home. Frontiers Architectural Research, 5, 351-352.

Altman, I. (1975). The Environment and Social Behavior: Privacy, Personal Space, Territory, and Crowding (First ed.). Monterey: Brooks/Cole.

American J. of Engineering and Applied Sciences (2010). The Influence of Modernity on Kurdish Architectural Identity,3(3), 554. Augustin, S., Frankel, N., \& Coleman, C. (2009). Place advantage: applied psychology for interior architecture (First ed.). Hoboken: John Wiley \& Sons.

Ayalp, N. (2012). Cultural Identity and Place Identity in House Environment: Traditional Turkish House Interiors. TOBB ETU University.

Bonnefoy, X. (2007). International Journal of Environment and Pollution. Inadequate housing and health,412.

Burris, A. (2014). Creature comforts: an exploration of comfort in the home. Lough-borough: Andrea Burris.

Butterworth, I. (2000). The Relationship between the Built Environment and Wellbeing (First ed.). Melbourne: Victorian Health Promotion Foundation.

Cheung, K. (1997). Design guide for interiors (First ed.). Omaha: US Army Corps of engineers.

Clayton, S. D. (2012). The Oxford handbook of environmental and conservation psychology. Oxford: Oxford University Press.

Eakin, G., \& Kurtich, J. (1993). The Interior Architecture (First ed.). New York: Van Nostrand Reinhold.

Einifar, A., \& Tabaeian, S. (2011). A Study of the Perspectives of Architectural and Environmental Psychology. International Journal of Architecture and Urban Development ,1(1), 28-31.

Ellis, R. (1986). Architects' People. (Third ed.). Arch. \& Compare.

Emamgholi, A. (2011). Flexible Spaces in Architecture. Khavaran: Higher-education Institute.

Fisher, S. (2015). Philosophy of Architecture. Stanford: The Stanford University Encyclopedia of Philosophy.

Georgiou, M. (2006). Architectural Privacy a Topological Approach to Relational Design Problems (First ed.). London: Bartlett School of Architecture.

Gifford, R., Steg, L., \& Reser, J. (2011). Environmental Psychology. Research Methods for Environmental Psychology, (18), 451.

Graetz,J. (2006). The Psychology of Learning Environment (First ed.). Winona: Winona State University.

Hall, E. T. (1969). The Hidden Dimension (First ed.). London: The Bodley Head.

Hall, E. T. (1990). The hidden dimension (Second ed.). Garden City: Edward T Hall.

Hauge, J (2009). Housing and Identity (First ed.). Trondheim: Norwegian University of Science and Technology.

Hauge, J. (2007). Architectural Science Review journal. Identity and place, (50), 1.

Heerwagen, J. (2006). Investing in People: The Social Benefits of Sustainable Design. In Rethinking Sustainable Construction (p. 4). Sarasota.

Hekkert, P. (2006). Design Aesthetics. Principle of Pleasure in Design. ,(48), 158-159.

Johnson, J. (2005). Embracing Complexity in Design (Third ed.). Paris: The Open University.

Karni, E. (2000). Movable Partitions. In Flexible Dwelling the Intersection of Dynamic Interior Space and Adaptable Dwelling Units (p. 179). ACSA.

Kearns, A., Hiscock, R., \& El laway, A. (n.d.). Beyond four walls. Housing Studies,15(3), 387.

Kim, J. (1998). Sustainable Architecture Module. National Pollution Prevention Center for Higher Education. Michigan: University of Michigan.

Koile, J. (2001). The Architect's Collaborator Toward Intelligent Tools for Conceptual Design (First ed.). Cambridge: Massachusetts institute of technology.

Marco, A., Stanley, K., \& Bluyssen, P. (2016). The 12th REHVA World Congress. In Energy consumption and Comfort in Homes (Vol. 6, p. 10). Aalborg University.

Moore, G. (1979). Architecture and Human Behavior: The Place of Environment-Behavior Studies in Architecture (First ed.). Madison: Wisconsin Architect.

Mothersill, P. (2014). The Form of Emotive Design (Master's thesis, Massachusetts Institute of Technology) (pp. 15-16). MIT Media Lab.

Namazian, A., \& Mehdipour, A. (2013). Psychological Demands of the Built Environment, Privacy, Personal Space and Territory in Architecture. Journal of Psychology and Behavioral Sciences ,3(4), 109-110.

Pallasmaa, J. (2014). Space, Place, and Atmosphere: Peripheral Perception in Existential Experience. Architectural Atmospheres,4, 230-245. doi:10.1515/9783038211785.18

Perolini, A. (2011). Interior Spaces and the Layers of Meaning. Design Principles \& Practices - An International Journal,5-6.

Petermans, A., \& Elisabeth, P. (2014). Design for subjective well-being in interior architecture. Annual Architectural Research Sympos in Finland,6, 207.

Razjoyan, M. (2015). Sound art and Architecture: New Horizons for Architecture and Urbanism. A look at the Combination of Architecture and Behavioral sciences,174, 3903-3908.

Richards, J. (1985). Hassan Fathy's concept of aesthetics in architecture (First ed.). London: Concept Media Pte Ltd.

Roessler, K. (2012). Healthy Architecture! Can environments evoke emotional responses. Canadian Center of Science and Education ,4(4), 83-84.

Serageldin, I. (2007). Much more than a building: reclaiming the legacy of the Bibliotheca Alexandrina (First ed.). Alexandria: Bibliotheca Alexandrina.

Sereda, P. J., \& Litvan, G. G. (1980). Durability of building materials and components: proceedings of the First International Conference: a symposium presented at Ottawa, Canada, 21-23 Aug. 1978(First ed.). Philadelphia: American Society for Testing and Materials.

Snyder, J., \& Catanese, A. (1979). Introduction to Architecture (First ed.). Madison: Wisconsin Architect.

Sorrento, L. (2012). A National Balance Interior Design, Humans, and Sustainability. Journal of Interior Design,2(37), 4-5. Wennberg, H. (2015). In Place a Study of Building\& Identity. Oxford: Seacourt Ltd. 